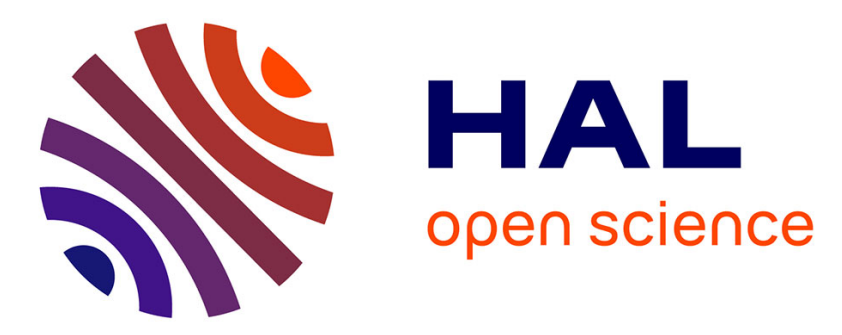

\title{
The Nash equilibrium region of the linear deterministic interference channel with feedback
}

\author{
Samir M. Perlaza, Ravi Tandon, H. Vincent Poor, Zhu Han
}

\section{To cite this version:}

Samir M. Perlaza, Ravi Tandon, H. Vincent Poor, Zhu Han. The Nash equilibrium region of the linear deterministic interference channel with feedback. 50th Allerton Conference on Communication, Control, and Computing (Allerton-2012), Oct 2012, Monticello, Illinois, United States. 10.1109/Allerton.2012.6483439 . hal-01280970

\section{HAL Id: hal-01280970 \\ https://hal.science/hal-01280970}

Submitted on 1 Mar 2016

HAL is a multi-disciplinary open access archive for the deposit and dissemination of scientific research documents, whether they are published or not. The documents may come from teaching and research institutions in France or abroad, or from public or private research centers.
L'archive ouverte pluridisciplinaire HAL, est destinée au dépôt et à la diffusion de documents scientifiques de niveau recherche, publiés ou non, émanant des établissements d'enseignement et de recherche français ou étrangers, des laboratoires publics ou privés. 


\title{
The Nash Equilibrium Region of the Linear Deterministic Interference Channel with Feedback
}

\author{
Samir M. Perlaza ${ }^{\dagger}$, Ravi Tandon ${ }^{\ddagger}$, H. Vincent Poor $^{\dagger}$ and Zhu Han ${ }^{\star}$. \\ ${ }^{\dagger}$ Department of Electrical Engineering. Princeton University, Princeton, NJ. \\ $\ddagger$ Department of Electrical and Computer Engineering. Virginia Tech, Blacksburg, VA \\ * Department of Electrical and Computer Engineering. University of Houston, Houston, TX. \\ \{perlaza, poor\}@princeton.edu, tandonr@vt.edu, zhan2@mail.uh.edu.
}

\begin{abstract}
The two-user linear deterministic interference channel (LD-IC) with feedback is studied under the assumption that each transmitter aims to selfishly maximize its individual achievable rate by tuning its own transmit configuration. More specifically, each transmitter autonomously tunes parameters such as the number of information bits per block, block length, codebook and encoding/decoding functions. To this end, the Nash equilibrium (NE) region of the LD-IC with feedback is fully characterized. The main observations presented in this paper are: $(i)$ The NE region with feedback is strictly larger than the NE region with no feedback. Moreover, all the new rate pairs achieved with feedback are either strictly Pareto or weakly Pareto optimal. (ii) The use of feedback allows the achievability of all Pareto optimal rate pairs of the capacity region of the LD-IC with feedback.
\end{abstract}

\section{INTRODUCTION}

Interference is an unavoidable phenomenon in wireless channels. The Gaussian interference channel (IC) is one of the fundamental building blocks of any wireless network. In this model transmitters aim to reliably transmit independent information to its respective receiver. Each receiver suffers additive interference from its unintended transmitters along with additive white Gaussian noise (AWGN). Even though the exact capacity region of the two-user Gaussian IC is unknown, significant progress has been made in this direction by studying the linear deterministic interference channel (LDIC) model [2]. In this model, the effects of the additive Gaussian noise are modeled by signal truncation, and the focus is on the interaction of the transmitted signals. It turns out that the LD-IC model captures the essential features of the Gaussian IC. Furthermore, insights gathered from the LD model have led to an approximate capacity characterization of the Gaussian IC in [2], [4], [6].

Since the transmitters in an interference channel are decentralized and are competing for the same resources, it is natural to expect a selfish behavior among the users to maximize their rates. This issue was studied by Berry and Tse in [3], in which the notion of a Nash equilibrium (NE) region was formulated and characterized for the LD interference channel. By using insights from the LD model, the Gaussian model was also investigated and the approximate NE region was characterized in [3]. In particular, a rate pair $\left(R_{1}\left(s_{1}, 2\right), R_{2}\left(s_{1}, s_{2}\right)\right)$ belongs to the $\mathrm{NE}$ region, if there exists a pair of transmit configurations (say $\left(s_{1}, s_{2}\right)$ ) satisfying

This research was supported in part by the Air Force Office of Scientific Research under MURI Grant FA9550-09-1-0643, and in part by the Army Research Office under MURI Grant W911NF-11-1-0036. the following properties: $(i)$ reliable information is sent at rate $R_{j}\left(s_{1}, s_{2}\right)$ to receiver $j, j=1,2$; $(i i)$ there exists no strategy $s_{1}^{\prime}$ such that $R_{1}\left(s_{1}^{\prime}, s_{2}\right)>R_{1}\left(s_{1}, s_{2}\right)$; and (iii) there exists no strategy $s_{2}^{\prime}$ such that $R_{2}\left(s_{1}, s_{2}^{\prime}\right)>R_{2}\left(s_{1}, s_{2}\right)$. If there exist a rate pair $\left(R_{1}\left(s_{1}, s_{2}\right), R_{2}\left(s_{1}, s_{2}\right)\right)$, such that the action pair $\left(s_{1}, s_{2}\right)$ satisfies the above three conditions, then the pair $\left(R_{1}\left(s_{1}, s_{2}\right), R_{2}\left(s_{1}, s_{2}\right)\right)$ is a Nash equilibrium. The set of all such NE rate pairs is the NE region and is denoted by $\mathcal{C}_{N E}$. Intuitively speaking, this notion implies that at any $\mathrm{NE}$, if a given user unilaterally deviates from the action pair $\left(s_{1}, s_{2}\right)$, it achieves a lower rate or equivalently, it would be unable to arbitrarily reduce its probability of error. It is also worth noting that the pair of strategies achieving a given rate pair in the $\mathrm{NE}$ region may not be unique, i.e., for a given $\left(R_{1}\left(s_{1}, s_{2}\right), R_{2}\left(s_{1}, s_{2}\right)\right) \in \mathcal{C}_{N E}$, there could be more than one distinct pairs of actions that achieve the same utility pair.

In this paper, we focus on the LD interference channel in which feedback is present from each receiver to its respective transmitter. It has recently been shown in [10] that feedback can provide substantial capacity gains in comparison when there is no feedback. In particular, feedback in the interference channel presents alternative paths for the information to reach the desired receivers. For instance, if one considers the Gaussian IC with strong interference, then an alternate path $T x_{1} \rightarrow R x_{2} \rightarrow T x_{2} \rightarrow R x_{1}$ can be used to achieve higher rates.

This observation motivates us to explore the usefulness of feedback under the scope of Nash equilibrium as studied in [3]. In particular, the fundamental questions of interest are how much feedback can help if users act in a selfish manner in order to maximize their utilities; and to what extent the alternative information paths created by feedback can be used. The main contribution of this paper is the complete characterization of the NE region of the two-user linear deterministic interference channel with feedback. Rather surprisingly, we show that the NE region in the presence of feedback is always larger than the NE region without feedback. Furthermore, in the strong interference regime, all points achievable with feedback satisfy the Nash equilibrium criteria, i.e., the feedback capacity region equals the NE region.

\section{Problem Formulation}

Consider a two-user interference channel. Transmitter $i$, with $i \in\{1,2\}$, communicates with its corresponding receiver by coding over blocks of length $N_{i}$ symbols. During channel use $t$, transmitter $i$ sends $L_{i}$ information bits $b_{i, 1}^{(t)}, \ldots, b_{i, L_{i}}^{(t)}$ 
by transmitting the codeword $\boldsymbol{x}_{i}^{(t)}=\left(x_{i, 1}^{(t)}, \ldots, x_{i, N_{i}}^{(t)}\right) \in \mathcal{C}_{i}$, where $\mathcal{C}_{i}$ denotes the codebook of transmitter $i$. Here, we assume that all information bits are independent and identically distributed following a uniform probability distribution. The input of receiver $i$ is denoted by $\boldsymbol{y}_{i}^{(t)}=\left(y_{i, 1}^{(t)}, \ldots, y_{i, N_{i}}^{(t)}\right)$. We assume a perfect feedback link from each receiver to its corresponding transmitter. Thus, at each channel use $t$, transmitter $i$ generates the codeword $\boldsymbol{x}_{i}^{(t)}$ based on its bits $b_{i, 1}^{(t)}, \ldots, b_{i, L_{i}}^{(t)}$ and all the previous sequences observed by its corresponding receiver $\boldsymbol{y}_{i}^{(1)}, \ldots, \boldsymbol{y}_{i}^{(t-1)}$. In this analysis, we also admit a random component (codeword) $\boldsymbol{w}_{i}^{(t)}$ to be taken into account to generate the codeword $\boldsymbol{x}_{i}^{(t)}$. We denote the codebook of random components by $\mathcal{W}_{i}$ and we assume that both transmitter $i$ and receiver $i$ have access to the source of random symbols. That is, both transmitter and receiver know the exact realization of their random component. The encoder of transmitter $i$ during channel use $t$ can be modeled as a deterministic mapping $f_{i}^{(t)}$ such that $\boldsymbol{x}_{i}^{(t)}=$ $f_{i}^{(t)}\left(k, \boldsymbol{y}_{i}^{(1)}, \ldots, \boldsymbol{y}_{i}^{(t-1)}, \boldsymbol{w}_{i}^{(t)}\right) \in \mathcal{C}_{i}$, where $k \in\left\{1, \ldots, 2^{L_{i}}\right\}$ is the index of the message to be transmitted.

At the end of the whole transmission, after channel use $T$, receiver $i$ uses the sequences $\boldsymbol{y}_{i}^{(1)}, \ldots, \boldsymbol{y}_{i}^{(T)}$ to generate estimates $\hat{b}_{i, \ell}^{(t)}$, for all $(\ell, t) \in\left\{1, \ldots, L_{i}\right\} \times\{1, \ldots, T\}$.

We denote the average bit error probability of transmitter $i$ during block $t$ by $p_{i}^{(t)}$ and we write,

$$
p_{i}^{(t)}=\frac{1}{L_{i}} \sum_{\ell=1}^{L_{i}} \mathbb{1}_{\left\{\hat{b}_{i, \ell}^{(t)} \neq b_{i, \ell}^{(t)}\right\}} .
$$

We say that the rate pair $\left(R_{1}, R_{2}\right) \in \mathbb{R}_{+}^{2}$ are achievable if there exists at least one pair of codebooks $\mathcal{C}_{1}$ and $\mathcal{C}_{2}$ with codewords of length $N_{1}$ and $N_{2}$, respectively, with the corresponding encoding functions $f_{1}$ and $f_{2}$ such that the average bit error probability can be made arbitrarily small by letting the block lengths $N_{1}$ and $N_{2}$ grow to infinity.

The aim of transmitter $i$ is to autonomously choose its transmit configuration $s_{i}$ in order to maximize its achievable rate $R_{i}$ given the transmit configuration $s_{j}$ of transmitter $j$. Here, the transmit configuration can be described in terms of the number of information bits per block $L_{i}$, the block length $N_{i}$, the codebook $\mathcal{C}_{i}$, the codebook of the random components $\mathcal{W}_{i}$ and the encoder function $f_{i}$. Note that the rate achieved by receiver $i$ depends on both configurations $s_{1}$ and $s_{2}$ due to the mutual interference naturally arising in the interference channel and due to feedback. In the following, we formulate this interaction by using the normal form formulation.

\section{A. Game Formulation}

The competitive interaction of both transmitters in the interference channel, as described in the previous section, can be modeled by the following game in normal-form:

$$
\mathcal{G}=\left(\mathcal{K},\left\{\mathcal{A}_{k}\right\}_{k \in \mathcal{K}},\left\{u_{k}\right\}_{k \in \mathcal{K}}\right) .
$$

Here, the set $\mathcal{K}=\{1,2\}$ is the set of players, that is, the set of transmitter-receiver pairs. The sets $\mathcal{A}_{1}$ and $\mathcal{A}_{2}$ are the sets of actions of player 1 and 2, respectively. An action of a player $i$, which we denote by $s_{i} \in \mathcal{A}_{i}$, is basically its transmit configuration. As previously mentioned, a transmit configuration is described in terms of all the parameters determining the coding/decoding scheme of the transmitter-receiver pair. The utility function of player $i$ is $u_{i}: \mathcal{A}_{1} \times \mathcal{A}_{2} \rightarrow \mathbb{R}_{+}$and we define it as the achieved rate of transmitter $i$,

$$
u_{i}\left(s_{1}, s_{2}\right)= \begin{cases}R_{1}\left(s_{1}, s_{2}\right), & \text { if } \quad \forall t \in\{1, \ldots, T\}, p_{i}^{(t)}<\epsilon, \\ 0, & \text { otherwise, }\end{cases}
$$

where $\epsilon>0$ is an arbitrarily small number and $R_{i}\left(s_{1}, s_{2}\right)$ denotes a transmission rate achievable with the configurations $s_{1}$ and $s_{2}$ such that $p_{i}^{(t)}<\epsilon$. Often, we refer to $R_{i}\left(s_{1}, s_{2}\right)$ as $R_{i}$ for the sake of simplicity. However, every non-negative rate is associated with a particular pair of transmit configurations $s_{1}$ and $s_{2}$. Similarly, we highlight the fact that there might exist several transmit configurations that achieve the same rate pair $\left(R_{1}, R_{2}\right)$.

In the following, we describe a particular class of action profiles $\boldsymbol{s}=\left(s_{1}, s_{2}\right) \in \mathcal{A}_{1} \times \mathcal{A}_{2}$ to which we refer as an $\eta$-Nash equilibrium ( $\eta$-NE).

Definition 1 ( $\eta$-Nash equilibrium): In the game $\mathcal{G}=$ $\left(\mathcal{K},\left\{\mathcal{A}_{k}\right\}_{k \in \mathcal{K}},\left\{u_{k}\right\}_{k \in \mathcal{K}}\right)$, an action profile $\left(s_{1}^{*}, s_{2}^{*}\right)$ is an $\eta$ Nash equilibrium if for all $i \in \mathcal{K}$ and for all $s_{i} \in \mathcal{A}_{i}$,

$$
u_{i}\left(s_{i}, s_{j}^{*}\right) \leqslant u_{i}\left(s_{i}^{*}, s_{j}^{*}\right)+\eta .
$$

From Def. 1, it becomes clear that if $\left(s_{1}^{*}, s_{2}^{*}\right)$ is an $\eta$ Nash equilibrium, then none of the transmitters can increase its transmission rate, while keeping the average bit error probability arbitrarily close to zero, by changing its own transmit configuration. Thus, at a given $\eta$-NE, every transmitter achieves a utility (transmission rate) that is $\eta$-close to its maximum achievable rate given the transmit configuration of the other transmitter. Note that if $\eta=0$, then the classical definition of Nash equilibrium (NE) is obtained [7]. The relevance of the notion of equilibrium is that at any $\mathrm{NE}$, every transmitter configuration is optimal with respect to the configuration of the other transmitters. In the following, we investigate the set of rate pairs that can be achieved at an NE. We refer to this set of rate pairs as the Nash region.

Definition 2 (Nash Region): An achievable rate pair $\left(R_{1}, R_{2}\right)$ is said to be in the Nash region of the game $\mathcal{G}=\left(\mathcal{K},\left\{\mathcal{A}_{k}\right\}_{k \in \mathcal{K}},\left\{u_{k}\right\}_{k \in \mathcal{K}}\right)$ if there exists an action profile $\left(s_{1}^{*}, s_{2}^{*}\right)$ that is an $\eta$-Nash equilibrium for an arbitrarily small $\eta$ and the following hold:

$$
u_{1}\left(s_{1}^{*}, s_{2}^{*}\right)=R_{1} \quad \text { and } \quad u_{2}\left(s_{1}^{*}, s_{2}^{*}\right)=R_{2} .
$$

In the following, we study the Nash region of the game $\mathcal{G}$ using a simple linearly deterministic approximation.

\section{LINEAR DETERMINISTIC MODELS}

In this section, we focus on a deterministic approximation model of the Gaussian interference channel, namely the linear deterministic IC. This model was first introduced in [1] and it has been widely accepted as a simple and insightful model to study the Gaussian inference channel. The linear deterministic IC is described by four parameters: $\left(n_{11}, n_{22}, n_{12}, n_{21}\right)$, where $n_{i i}$ captures the signal strength from transmitter $i$ to receiver $i$, and $n_{i j}$ captures the interference strength from transmitter $j$ to receiver $i$. The input-output relationship is given as follows:

$$
\begin{aligned}
& Y_{1}(t)=S^{q-n_{11}} X_{1}(t)+S^{q-n_{12}} X_{2}(t), \text { and } \\
& Y_{2}(t)=S^{q-n_{21}} X_{1}(t)+S^{q-n_{22}} X_{2}(t),
\end{aligned}
$$


where $X_{i}(t), Y_{i}(t) \in\{0,1\}^{q}$, with $q=\max _{(i, j) \in\{1,2\}^{2}} n_{i j}$, addition/multiplication are over a binary field, and $S$ is a $q \times q$ shift matrix

$$
S=\left[\begin{array}{cccc}
0 & 0 & \cdots & 0 \\
1 & 0 & \ddots & 0 \\
0 & 1 & \ddots & 0 \\
\vdots & \vdots & \ddots & 0 \\
0 & 0 & \cdots & 1
\end{array}\right]
$$

We note that $n_{i i}$ corresponds to $\log _{2}\left(\mathrm{SNR}_{i}\right)$ and $n_{j i}$ corresponds to $\log _{2}\left(\mathrm{INR}_{j i}\right)$, where $\mathrm{SNR}_{i}$ is the signal-to-noise ratio at receiver $i$ and $\mathrm{INR}_{j i}$ is the interference-to-noise ratio at receiver $j$ from transmitter $i$ in the corresponding Gaussian interference channel. For a detailed discussion of the connection of the LD-IC to the Gaussian IC, we refer the reader to [4].

In the following, we describe some of the known results about the LD-IC with and without feedback from which we build up our main contributions.

\section{A. Capacity of the LD-IC without Feedback}

We denote by $\mathcal{C}$ the capacity region of the two-user LDIC without feedback. The region $\mathcal{C}$ is fully characterized by Lemma 4 in [4]. We reproduce this result for the sake of completeness.

Lemma 1 (Capacity Region of the LD-IC w/o FB [4]):

The capacity region $\mathcal{C}$ of the $L D-I C$ without feedback is given by the set of non-negative rate pairs satisfying

$$
\begin{array}{cc}
R_{i} \leqslant & n_{i i}, \text { with } i \in\{1,2\}, \\
R_{1}+R_{2} \leqslant & \left(n_{11}-n_{12}\right)^{+}+\max \left(n_{22}, n_{12}\right), \\
R_{1}+R_{2} \leqslant & \left(n_{22}-n_{21}\right)^{+}+\max \left(n_{11}, n_{21}\right), \\
R_{1}+R_{2} \leqslant & \max \left(n_{21},\left(n_{11}-n_{12}\right)^{+}\right. \\
& +\max \left(n_{12},\left(n_{22}-n_{21}\right)^{+},\right. \\
2 R_{1}+R_{2} \leqslant & \max \left(n_{11}, n_{21}\right)+\left(n_{11}-n_{12}\right)^{+} \\
& +\max \left(n_{12},\left(n_{22}-n_{21}\right)\right)^{+}, \\
R_{1}+2 R_{2} \leqslant & \max \left(n_{22}, n_{12}\right)+\left(n_{22}-n_{21}\right)^{+}, \\
& +\max \left(n_{21},\left(n_{11}-n_{12}\right)\right)^{+} .
\end{array}
$$

Note that the capacity region shown in Lemma 1 is a particular case of the capacity region presented in [5] that applies to a larger class of deterministic interference channels.

\section{B. Nash Region of the LD-IC without Feedback}

The Nash region of the linear deterministic interference channel without feedback has been fully characterized in [3]. Here, we denote this region by $\mathcal{C}_{\mathrm{NE}}$. In order to formally define $\mathcal{C}_{\mathrm{NE}}$, we first introduce the following set:

$$
\mathcal{B}=\left\{\left(R_{1}, R_{2}\right) \in \mathbb{R}^{2}: L_{i} \leqslant R_{i} \leqslant U_{i}, \forall i \in\{1,2\}\right\},
$$

where, $\forall i \in\{1,2\}$,

$$
\begin{aligned}
L_{i} & =\left(n_{i i}-n_{i j}\right)^{+}, \\
U_{i} & =\left\{\begin{array}{lll}
n_{i i}-\min \left(L_{j}, n_{i j}\right) & \text { if } & n_{i j} \leqslant n_{i i} \\
\min \left(\left(n_{i j}-L_{j}\right)^{+}, n_{i i}\right) & \text { if } & n_{i j}>n_{i i} .
\end{array}\right.
\end{aligned}
$$

Then, from Theorem 1 in [3], we define $\mathcal{C}_{\mathrm{NE}}$ using the following lemma.

Lemma 2 (Nash Region of the LD-IC w/o FB [3]): Let $\mathcal{C}_{\mathrm{NE}}$ denote the Nash region of the LD-IC without feedback. Then,

$$
\mathcal{C}_{\mathrm{NE}}=\mathcal{B} \cap \mathcal{C}
$$

\section{Capacity of the LD-IC with Feedback}

We denote the capacity of the linear deterministic interference channel with feedback by $\mathcal{C}_{\mathrm{FB}}$. From Corollary 1 in [10], we have the following lemma.

Lemma 3 (Capacity Region of the LD-IC with FB [10]): The capacity region $\mathcal{C}_{\mathrm{FB}}$ of the $L D-I C$ with feedback is given by the set of non-negative rate pairs satisfying

$$
\begin{aligned}
& R_{1} \leqslant \min \left(\max \left(n_{11}, n_{12}\right), \max \left(n_{11}, n_{21}\right)\right), \\
& R_{2} \leqslant \min \left(\max \left(n_{22}, n_{21}\right), \max \left(n_{22}, n_{12}\right)\right), \\
& R_{1}+R_{2} \leqslant \min \left(\max \left(n_{22}, n_{12}\right)+\left(n_{11}-n_{12}\right)^{+},\right. \\
&\left.\left.\max \left(n_{11}, n_{21}\right)+\left(n_{22}-n_{21}\right)^{+}\right)\right\} .
\end{aligned}
$$

\section{Main Results}

We denote the Nash region of the LD-IC with feedback by $\mathcal{C}_{\mathrm{FB} / \mathrm{NE}}$. In order to define the set $\mathcal{C}_{\mathrm{FB} / \mathrm{NE}}$, we first introduce the following set:

$$
\mathcal{B}_{\mathrm{FB}}=\left\{\left(R_{1}, R_{2}\right) \in \mathbb{R}^{2}: R_{i} \geqslant L_{i}, \forall i \in\{1,2\}\right\},
$$

where $L_{i}$ is defined in (8). We state our main result for the linear deterministic IC with feedback in terms of the set $\mathcal{B}_{\mathrm{FB}}$.

Theorem 1: For a two-user linear deterministic IC with feedback,

$$
\mathcal{C}_{\mathrm{FB} / \mathrm{NE}}=\mathcal{B}_{\mathrm{FB}} \cap \mathcal{C}_{\mathrm{FB}}
$$

In order to illustrate this result, we consider a symmetric linear deterministic IC with feedback in which, $n=n_{11}=n_{22}$ and $m=n_{12}=n_{21}$, with normalized cross gain $\alpha=\frac{m}{n}$. In Fig. 1, we plot $\mathcal{C}, \mathcal{C}_{\mathrm{NE}}, \mathcal{C}_{\mathrm{FB}}$, and $\mathcal{C}_{\mathrm{FB} / \mathrm{NE}}$ for different interference regimes, i.e., very weak interference $\left(0 \leqslant \alpha \leqslant \frac{1}{2}\right)$, weak interference $\left(\frac{1}{2}<\alpha \leqslant \frac{2}{3}\right)$, moderate interference $\left(\frac{2}{3}<\alpha \leqslant 1\right)$, strong interference $(1<\alpha \leqslant 2)$ and very strong interference $(\alpha \geqslant 2)$, respectively. Note that all the rates pairs $\left(R_{1}, R_{2}\right)$, such that $R_{1}>L_{1}$ and $R_{2}>L_{2}$, are achievable at an NE when feedback is used. This observation underlines the main benefits of using feedback when all users act selfishly. For instance, in all the interference regimes, the use of feedback increases the number of rate pairs that are achievable at the NE with respect to the case without feedback. More importantly, the new achievable rate pairs are either Pareto optimal or weakly Pareto superior to the rate pairs achievable without feedback at the NE. More precisely, for all $\left(R_{1}^{*}, R_{2}^{*}\right) \in \mathcal{C}_{\mathrm{NE}}$ there always exists a rate pair $\left(R_{1}^{+}, R_{2}^{+}\right) \in \mathcal{C}_{\mathrm{FB} / \mathrm{NE}}$ such that $R_{i}^{+} \geqslant R_{i}^{*}$, for all $i \in\{1,2\}$. This observation confirms that in this setup, increasing the space of actions by letting users use feedback does not decrease neither their individual rates nor the aggregated achievable transmission rate. Note that the converse effect has been observed in the parallel IC and parallel multiple access channel (MAC) for particular scenarios in [8] and [9]. This reasoning can be summarized by 

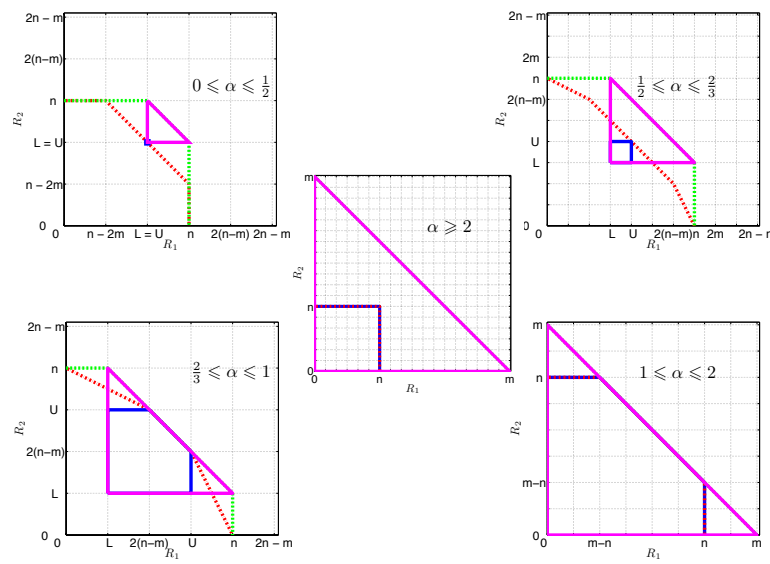

Fig. 1. Illustration of $\mathcal{C}$ (red dotted line), $\mathcal{C}_{\mathrm{NE}}$ (solid blue line), $\mathcal{C}_{\mathrm{FB}}$ (green dotted line), and $\mathcal{C}_{\mathrm{FB} / \mathrm{NE}}$ (magenta solid line) in all interference regimes.

writing the following inclusion that holds with strict inequality for all regimes:

$$
\mathcal{C}_{\mathrm{NE}} \subset \mathcal{C}_{\mathrm{FB} / \mathrm{NE}}
$$

However, the main advantage of using feedback when users act selfishly is that it allows the achievability of all Pareto optimal rate pairs of $\mathcal{C}_{\mathrm{FB}}$. That is, all the sum-rate maximizing rate pairs in $\mathcal{C}_{\mathrm{FB}}$ are achievable at the $\mathrm{NE}$ in all the interference regimes. It is worth noting that in the case $\alpha>1$ with feedback, $\mathcal{C}_{\mathrm{FB}} \subset \mathcal{B}_{\mathrm{FB}}$ and thus, all the achievable rates of the IC with feedback are also achievable at the NE, i.e., $\mathcal{C}_{\mathrm{FB} / \mathrm{NE}}=\mathcal{C}_{\mathrm{FB}}$. This contrasts with the case without feedback, in which only a subset of the Pareto optimal rate pairs in $\mathcal{C}$ are achievable at the equilibrium in most of the interference regimes. For instance, when $0 \leqslant \alpha \leqslant \frac{1}{2}$ and $\frac{1}{2}<\alpha \leqslant \frac{2}{3}$ (see Fig. 1) only one pair $\left(R_{1}, R_{2}\right)$ of the infinitely many Pareto optimal pairs is achieved at the equilibrium.

In the following, we present a few examples to provide some intuitions into the impact of feedback on the Nash equilibrium region of the $\mathrm{IC}$ with feedback.

\section{A. Examples}

Consider the scenario of very weak interference, for instance, let $\alpha=\frac{1}{3}$, with $m=2$ and $n=6$. From Theorem 1 , it follows that the Nash region is $\mathcal{C}_{\mathrm{FB} / \mathrm{NE}}=\left\{\left(R_{1}, R_{2}\right) \in\right.$ $\left.\mathbb{R}^{2}: \forall i R_{i} \geqslant 4, R_{1}+R_{2} \leqslant 10\right\}$. In Fig. 1 , the region $\mathcal{C}_{\mathrm{FB} / \mathrm{NE}}$ corresponds to the convex hull of the points $(4,4),(6,4)$ and $(4,6)$. In the following, we show the pair $(4,4)$ is achieved without feedback, the rate pair $(6,4)$ or $(4,6)$ is achieved when one of the transmitters uses feedback and the rate pair $(5,5)$ is achieved when both players use feedback.

a) Achievability of $(4,4)$ : The rate pair $(4,4)$ is achievable when none of the transmitters uses feedback (See Fig. 1). Note that when one of the transmitters sends only private messages to its corresponding receiver, the highest achievable rate of the other transmitter is achieved by sending only private messages to its corresponding receiver. Here, any attempt of a transmitter to increase its rate by using its $m$ lowest levels would bound its probability of error away from zero since those levels are subject to the interference of the $m$ highest levels of the other transmitter. That is, when both players send
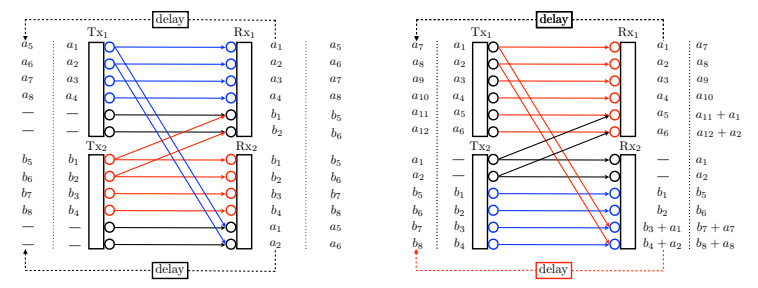

(a)

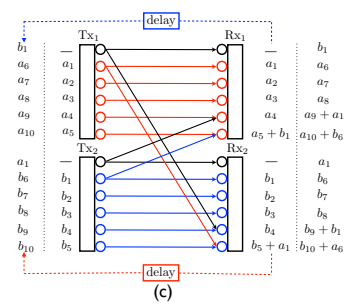

(b)

Fig. 2. Coding scheme for achieving the rate pair $(4,4)$ in $(a),(6,4)$ in (b) and $(5,5)$ in $(\mathrm{c})$ at the NE.

new bits at every channel use using their $n-m$ highest levels (see Fig. 2a), this configuration is an NE independently of whether one or both transmitters implement feedback. In this case, the bits obtained at the transmitter via feedback are not useful to improve its own coding/decoding scheme.

The choice of using all the highest levels to transmit new bits at every channel use, or equivalently not using feedback, can be associated with a greedy behavior. As we shall see in the next example, if one of the transmitters uses feedback it would obtain the same rate but it would allow the other transmitter to achieve a higher rate.

b) Achievability of $(6,4)$ and $(4,6)$ : The rate pairs $(6,4)$ and $(4,6)$ are achievable at an NE when one of the transmitters uses feedback. Consider for instance that transmitter 1 uses all its $n$ levels to transmit new information at each channel use, that is, it does not use feedback. Under this condition, the maximum rate achievable by transmitter 2 is 4 bits per channel use (see the capacity region $\mathcal{C}_{\mathrm{FB}}$ ). Note that the rate of 4 bits per channel use can be achieved by transmitter 2 by simply using its $n-m$ highest levels or using its $n-m$ lowest levels with feedback to resolve the interference produced by the top levels of transmitter 1 . In both cases, any attempt by transmitter 2 to use its $m$ highest levels to send new bits instead of those obtained via feedback would constraint transmitter 1 to achieve a rate of 6 bits per channel use. This is basically because at least $m$ bits would not be decoded reliably at receiver 1 . Thus, the rate pair $(6,4)$ is an NE if there exists a coding scheme for transmitter 2 that does not use its $m$ highest levels and achieves at least a rate of 4 bits per channel use. This coding scheme is presented in Fig. $2 b$.

Note that transmitter 2 uses its $n-m$ lowest levels to transmit new information at each channel use, and thus, at least $m$ of these bits are subject to the interference of the $m$ highest levels of transmitter 1 . However, by using feedback, transmitter 2 can transmit over its $m$ highest levels during channel use $t$, the $m$ symbols received by its $m$ lowest levels in channel use $t-1$. At channel use $t$, these retransmitted symbols $\left(s_{1}\right.$ and $s_{2}$ in Fig. 2b) are received in the $m$ lowest levels of receiver 1 , however, they do not represent any interference as they have been decoded in the previous channel use $t-1$ and thus, they can be subtracted. At receiver 2, these bits are received interference-free at the $m$ highest levels during channel use $t$ and can be used to decode the $m$ bits received 
in the $m$ lowest levels in the previous block $t-1$. Thus, this coding scheme achieves a rate of 4 bits per channel use without using the $m$ highest levels of transmitter 2. This shows that the rate pair $(6,4)$ can be achieved as an NE. By exchanging the identity of the players in the analysis above, we show the achievability of the rate pair $(4,6)$.

In general, it is worth noting that when player $i$ implements a strategy that does not use $s \leqslant m$ of its highest levels for transmitting new bits but rather those obtained via feedback, transmitter $i$ grants $s$ additional bits per channel use to transmitter $j$ compared to the case when it does not perform feedback. That is, transmitter $j$ achieves a rate $R_{j}=(n-m)^{+}+s$ and transmitter $i$ achieves $R_{i}=(n-s)^{+}$. In both cases, both transmitters achieve a rate which is at least equal to that achieved without feedback. Moreover, it is easy to verify that neither of the transmitters can increase its transmission rate by unilateral deviation and thus, these rate pairs are achievable at an NE.

The underlying conclusion of this example is that a transmitter using feedback does not negatively affect its own transmission rate but significantly benefits the other transmitter. Thus, we often associate the use of feedback with an altruistic behavior.

We analyze the case in which both transmitters implement feedback in the following example.

c) Achievability of $(5,5)$ : The rate pair $(5,5)$ is achieved at an NE when both transmitters use feedback. In this case, both transmitters use their highest levels to transmit the bits obtained via feedback instead of new bits at every channel use. The coding scheme that achieves this rate pair is presented in Fig. 2c. Therein, it can be verified that any attempt by either of the transmitters to increase its individual rate by sending new bits at each channel use (instead of those obtained via feedback) raises its own probability of error. This is basically because at least one bit would never be reliably decoded. This verifies that the rate pair $(5,5)$ is achievable at an NE.

\section{PROOFS}

To prove Theorem 1 , we first show that a rate pair $\left(R_{1}, R_{2}\right)$, with $R_{i} \leqslant\left(n_{i i}-n_{i j}\right)^{+}$for at least one $i \in\{1,2\}$ is not an $\eta$-equilibrium for an arbitrarily small $\eta$. That is,

$$
\mathcal{C}_{\mathrm{FB} / \mathrm{NE}} \subseteq \mathcal{C}_{\mathrm{FB}} \cap \mathcal{B}_{\mathrm{FB}}
$$

Later, we show that any point in $\mathcal{C}_{\mathrm{FB}} \cap \mathcal{B}_{\mathrm{FB}}$ is an $\eta$-equilibrium for all $\eta>0$. That is,

$$
\mathcal{C}_{\mathrm{FB} / \mathrm{NE}} \supseteq \mathcal{C}_{\mathrm{FB}} \cap \mathcal{B}_{\mathrm{FB}}
$$

which proves Theorem 1 .

We start by stating the following lemma.

Lemma 4: A rate pair $\left(R_{1}, R_{2}\right) \in \mathcal{C}_{\mathrm{FB}}$, with either $R_{1}<$ $\left(n_{11}-n_{12}\right)^{+}$or $R_{2}<\left(n_{22}-n_{21}\right)^{+}$is not an $\eta$-equilibrium, with $\eta>0$.

Proof: Let $\left(s_{1}, s_{2}\right)$ be an action profile such that users achieve the rate pair $R_{1}\left(s_{1}, s_{2}\right)$ and $R_{2}\left(s_{1}, s_{2}\right)$, respectively. Assume, without any loss of generality, that $R_{1}\left(s_{1}, s_{2}\right)<$ $\left(n_{11}-n_{12}\right)^{+}$. Then, note that there exists at least one action $s_{1}^{\prime}$, such that transmitter 1 uses its top levels, which are interference free, and thus it is always able to achieve a rate $R_{1}\left(s_{1}^{\prime}, s_{2}\right) \geqslant\left(n_{11}-n_{12}\right)^{+}$. Note also that the utility improvement $R_{1}\left(s_{1}^{\prime}, s_{2}\right)-R_{1}\left(s_{1}, s_{2}\right)>0$ is always possible independently of the current action $s_{2}$ of user 2. Thus, it follows that the action profile $\left(s_{1}, s_{2}\right)$ is not an $\eta$-equilibrium, for an arbitrarily small $\eta$, which completes the proof.

To continue with our proof, we introduce a modification to the feedback coding scheme presented in [10]. The novelty, with respect to [10], consists of allowing users to introduce some random symbols into their common messages as done in [3] for the case of the IC without feedback. At each block $b$ transmitter $i$ divides its message $w_{i}^{(b)}$ into two parts: a private part $w_{i p}^{(b)}$ and a common part $w_{i c}^{(b)}$ such that $w_{i}^{(b)}=\left(w_{i p}^{(b)}, w_{i c}^{(b)}\right)$. The private message $w_{i p}^{(b)} \in\left\{1, \ldots, 2^{n R_{i p}}\right\}$ is sent in the $\left(n_{i i}-n_{j i}\right)^{+}$less significant levels of transmitter $i$ and thus, it is seen only by receiver $i$. The common message is sent in the first $n_{j i}$ most significant levels of transmitter $i$ and thus, it is seen by both receivers. The common message index $w_{i c}^{(b)}$ is determined based on the actual common message index $m_{i c}^{(b)} \in\left\{1, \ldots, 2^{n R_{i c}}\right\}$ and a randomly generated symbol $m_{i r}^{(b)} \in\left\{1, \ldots, 2^{n R_{i r}}\right\}$. Transmitter $i$ uses a mapping $f_{i}:\left\{1, \ldots, 2^{n R_{i c}}\right\} \times\left\{1, \ldots, 2^{n R_{i r}}\right\} \rightarrow\left\{1, \ldots, 2^{n\left(R_{i r}+R_{i c}\right)}\right\}$, such that $f_{i}\left(m_{i c}^{(b)}, m_{i r}^{(b)}\right)=w_{i c}^{(b)}$. The random symbols are assumed to be known at the receiver $i$ and thus, the index $m_{i r}^{(b)}$ conveys no information to either receiver. That is, if transmitter $i$ achieves a rate pair $\left(R_{i p}, R_{i c}, R_{i r}\right)$, its actual rate is $R_{i}=R_{i c}+R_{i p}$. In particular, we assume that the random common messages are selected uniformly from the set $\left\{1, \ldots, 2^{n R_{i r}}\right\}$ and we refer to this coding scheme as a randomized coding scheme with feedback. We describe this coding scheme as follows:

Codebook Generation: Fix a joint probability distribution $p\left(u, u_{1}, u_{2}, x_{1}, x_{2}\right) \quad=$ $p(u) p\left(u_{1} \mid u\right) p\left(u_{2} \mid u\right) p\left(x_{1} \mid u, u_{1}\right) p\left(x_{2} \mid u, u_{2}\right) . \quad$ Generate $2^{n\left(R_{1 c}+R_{1 r}+R_{2 c}+R_{2 r}\right)}$ independent and identically distributed (i.i.d.) length- $n$ codewords $\left.\boldsymbol{u}(s, t)=\underset{n}{\left(u_{1}\right.}(s, t), \ldots, u_{n}(s, t)\right)$ according to $p(\boldsymbol{u}(s, t))=\prod_{m=1}^{n} p\left(u_{m}(s, t)\right)$, with $s \in\left\{1, \ldots, 2^{n\left(R_{1 c}+R_{1 r}\right)}\right\}$ and $t \in\left\{1, \ldots, 2^{n\left(R_{2 c}+R_{2 r}\right)}\right\}$.

For encoder 1, generate for each codeword $\boldsymbol{u}(s, t), \quad 2^{n\left(R_{1 c}+R_{1 r}\right)} \quad$ i.i.d. length- $n$ codewords $\boldsymbol{u}_{1}(s, t, k)=\left(u_{1,1}(s, t, k), \ldots, u_{1, n}(s, t, k)\right)$ according to $p\left(\boldsymbol{u}_{1}(s, t, k) \mid \boldsymbol{u}(s, t)\right)=\prod_{m=1}^{n} p\left(u_{1, m}(s, t, k) \mid u_{m}(s, t)\right)$, with $k \in\left\{1, \ldots, 2^{n\left(R_{1 c}+R_{1 r}\right)}\right\}$. For each pair of codewords $\left(\boldsymbol{u}(s, t), \boldsymbol{u}_{1}(s, t, k)\right), \quad$ generate $\quad 2^{n\left(R_{1 p}\right)} \quad$ i.i.d. length- $n$ codewords $\boldsymbol{x}_{1}(s, t, k, l)=\left(x_{1,1}(s, t, k, l), \ldots, x_{1, n}(s, t, k, l)\right)$ according to $p\left(\boldsymbol{x}(s, t, k, l) \mid \boldsymbol{u}(s, t), \boldsymbol{u}_{1}(s, t, k)\right)=$ $\prod_{m=1}^{n} p\left(x_{1, m}(s, t, k, l) \mid u_{m}(s, t), u_{1, m}(s, t, k)\right), \quad$ with $l \in\left\{1, \ldots, 2^{n R_{1 p}}\right\}$.

For encoder 2, generate for each codeword $\boldsymbol{u}(s, t), \quad 2^{n\left(R_{2 c}+R_{2 r}\right)} \quad$ i.i.d. length- $n$ codewords $\boldsymbol{u}_{2}(s, t, q)=\left(u_{2,1}(s, t, q), \ldots, u_{2, n}(s, t, q)\right)$ according to $p\left(\boldsymbol{u}_{2}(s, t, q) \mid \boldsymbol{u}(s, t)\right)=\prod_{m=1}^{n} p\left(u_{2, m}(s, t, q) \mid u_{m}(s, t)\right)$, with $q \in\left\{1, \ldots, 2^{n\left(R_{2 c}+R_{2 r}\right)^{m}}\right\}$. For each pair of codewords $\left(\boldsymbol{u}(s, t), \boldsymbol{u}_{2}(s, t, q)\right), \quad$ generate $2^{n\left(R_{2 p}\right)} \quad$ i.i.d. length- $n$ 
codewords $\boldsymbol{x}_{2}(s, t, q, z)=\left(x_{2,1}(s, t, q, z), \ldots, x_{2, n}(s, t, q, z)\right)$ according to $p\left(\boldsymbol{x}_{2}(s, t, k, l) \mid \boldsymbol{u}(s, t), \boldsymbol{u}_{2}(s, t, k)\right)=$ $\prod_{m=1}^{n} p\left(x_{2, m}(s, t, k, l) \mid u_{m}(s, t), u_{2, m}(s, t, q)\right)$,

with $z \in\left\{1, \ldots, 2^{n R_{2 p}}\right\}$.

Encoding: We follow a superposition coding scheme. At block $b=1$, transmitter $i$ sends the symbol $\boldsymbol{x}_{i}^{(1)}=\boldsymbol{x}_{i}\left(s^{*}, t^{*}, w_{i c}^{(1)}, w_{i p}^{(1)}\right)$, where both $s^{*}$ and $t^{*}$ are predefined indices known at both transmitters and receivers. At block $b>1$, transmitter 1 sends the codeword $\boldsymbol{x}_{1}^{(b)}=\boldsymbol{x}_{1}\left(w_{1 c}^{(b-1)}, w_{2 c}^{(b-1)}, w_{1 c}^{(b)}, w_{1 p}^{(b)}\right)$, where $w_{2 c}^{(b-1)}$ is obtained from the feedback of $\boldsymbol{y}_{1}^{(b-1)}$ at the end of block $b-1$. That is, $w_{2 c}^{(b-1)}=\hat{t}$, with $\hat{t} \in\left\{1, \ldots, 2^{n\left(R_{2 c}+R_{2 r}\right)}\right\}$, if $\hat{t}$ is the only index that satisfies

$$
\begin{aligned}
& \left(\boldsymbol{u}\left(w_{1 c}^{(b-2)}, w_{2 c}^{(b-2)}\right), \boldsymbol{u}_{1}\left(w_{1 c}^{(b-2)}, w_{2 c}^{(b-2)}, w_{1 c}^{(b-1)}\right),\right. \\
& \boldsymbol{x}_{1}\left(w_{1 c}^{(b-2)}, w_{2 c}^{(b-2)}, w_{1 c}^{(b-1)}, w_{1 c}^{(b-1)}\right), \\
& \left.\boldsymbol{u}_{2}\left(w_{1 c}^{(b-2)}, w_{2 c}^{(b-2)}, \hat{t}\right), \boldsymbol{y}_{1}^{(b-1)}\right) \in A_{e}^{(n)},
\end{aligned}
$$

under the assumption that $w_{2 c}^{(1)}, \ldots, w_{2 c}^{(b-2)}$ have been decoded without errors at transmitter 1 . At block $B$, transmitter 1 sends the symbol $x_{1}^{(B)}=x_{1}\left(w_{1 c}^{(B-1)}, w_{2 c}^{(B-1)}, k^{*}, l^{*}\right)$, where both $k^{*}$ and $l^{*}$ are predefined indices known at both transmitters and the receiver. Transmitter 2 follows a similar encoding scheme. Decoding: Both receivers decode their messages at the end of the $B$ blocks in a backward decoding fashion. At each block $b$, with $b=1$ denoting the last received block, receiver 1 determines the unique pair of message indices $(\hat{s}, \hat{t}, \hat{k}) \in$ $\left\{1, \ldots, 2^{n\left(R_{1 c}+R_{1 r}\right)}\right\} \times\left\{1, \ldots, 2^{n\left(R_{2 c}+R_{2 r}\right)}\right\} \times\left\{1, \ldots, 2^{n R_{1 p}}\right\}$ that satisfies

$$
\begin{aligned}
& \left(\boldsymbol{u}(\hat{s}, \hat{t}), \boldsymbol{u}_{1}\left(\hat{s}, \hat{t}, w_{1 c}^{(b)}\right), \boldsymbol{x}_{1}\left(\hat{s}, \hat{t}, w_{1 c}^{(b)}, \hat{k}\right),\right. \\
& \left.\boldsymbol{u}_{2}\left(\hat{s}, \hat{t}, w_{2 c}^{(b)}\right), \boldsymbol{y}_{1}^{(b)}\right) \in A_{e}^{(n)}
\end{aligned}
$$

where $w_{1 c}^{(1)}=w_{2 c}^{(1)}=k^{*}$ and $w_{1 p}^{(1)}=w_{2 p}^{(1)}=l^{*}, w_{1 c}^{(B)}=$ $w_{2 c}^{(B)}=s^{*}$ and $w_{1 p}^{(B)}=w_{2 p}^{(B)}=t^{*}$.

Receiver 2 follows a similar decoding scheme.

Probability of Error Analysis: An error might occur during the coding phase if the message $w_{j c}^{(b)}$ is not correctly decoded at transmitter $i$ at the end of block $b$. For instance, this error might occur at transmitter 1 because: $(i)$ there does not exist an index $\hat{t} \in\left\{1, \ldots, 2^{n\left(R_{2 c}+R_{2 r}\right)}\right\}$ that satisfies (17), or (ii) several indices simultaneously satisfy (17). From the asymptotic equipartion property (AEP), the probability of error due to $(i)$ tends to zero when $n$ grows to infinity. The probability of error due to $(i i)$ can be made arbitrarily close to zero when $n$ grows to infinity, if

$$
R_{i c}+R_{i r} \leqslant I\left(U_{i} ; Y_{j} \mid X_{j}, U\right) .
$$

An error might occur during the decoding phase of block $b$ if the messages $w_{1 c}^{(b-1)}, w_{2 c}^{(b-1)}$ and $w_{1 p}^{(b)}$ are not decoded correctly. These errors might arise due to two reasons: $(i)$ there does not exist a pair $(\hat{s}, \hat{t}, \hat{k})$ that satisfies (18), or $(i i)$ there exist several pairs $(\hat{s}, \hat{t}, \hat{k})$ that simultaneously satisfy (18). From the AEP, the probability of an error due to $(i)$ tends to zero when $n$ tends to infinity. We focus now on the error due to $(i i)$. Define the following event during the decoding interval of block $b$,

$$
\begin{aligned}
E_{s t k}^{(b)}= & \left\{\left(\boldsymbol{u}(s, t), \boldsymbol{u}_{1}\left(s, t, w_{1 c}^{(b)}\right), \boldsymbol{x}_{1}\left(s, t, w_{1 c}^{(b)}, k\right),\right.\right. \\
& \left.\left.\boldsymbol{u}_{2}\left(\hat{s}, \hat{t}, w_{2 c}^{(b)}\right), \boldsymbol{y}_{1}^{(b)}\right) \in A_{e}^{(n)}\right\} .
\end{aligned}
$$

Assume also that at block $b$ the indices $(\hat{s}, \hat{t}, \hat{k})$ are $(1,1,1)$ without any loss of generality, due to the symmetry of the code. Then, the probability of error due to $(i i)$ during block $b, P_{e}^{(b)}$, can be bounded as follows:

$$
\begin{aligned}
P_{e}^{(b)}= & \operatorname{Pr}\left(\bigcup_{(s, t, k) \neq(1,1,1)} E_{s t k}^{(b)}\right) \\
\leqslant & \sum_{s \neq 1, t \neq 1, k \neq 1} \operatorname{Pr}\left(E_{s t k}^{(b)}\right)+\sum_{s=1, t \neq 1, k \neq 1} \operatorname{Pr}\left(E_{s t k}^{(b)}\right) \\
& +\sum_{s \neq 1, t=1, k \neq 1} \operatorname{Pr}\left(E_{s t k}^{(b)}\right)+\sum_{s \neq 1, t \neq 1, k=1} \operatorname{Pr}\left(E_{s t k}^{(b)}\right) \\
& +\sum_{s=1, t=1, k \neq 1} \operatorname{Pr}\left(E_{s t k}^{(b)}\right)+\sum_{s \neq 1, t=1, k=1} \operatorname{Pr}\left(E_{s t k}^{(b)}\right) \\
& +\sum_{s=1, t \neq 1, k=1} \operatorname{Pr}\left(E_{s t k}^{(b)}\right) \\
\leqslant & 2^{n\left(R_{1 c}+R_{1 r}+R_{2 c}+R_{2 r}+R_{1 p}-I\left(U, U_{2}, X_{1} ; Y_{1}\right)+4 \epsilon\right)} \\
& +2^{n\left(R_{2 c}+R_{2 r}+R_{1 p}-I\left(U, U_{2}, X_{1} ; Y_{1}\right)+4 \epsilon\right)} \\
& +2^{n\left(R_{1 c}+R_{1 r}+R_{1 p}-I\left(U, U_{2}, X_{1} ; Y_{1}\right)+4 \epsilon\right)} \\
& +2^{n\left(R_{1 c}+R_{1 r}+R_{2 c}+R_{2 r}-I\left(U, U_{2}, X_{1} ; Y_{1}\right)+4 \epsilon\right)} \\
& +2^{\left.n R_{1 p}-I\left(X_{1} ; Y_{1} \mid U, U_{1}, U_{2}\right)+4 \epsilon\right)} \\
& +2^{n\left(R_{1 c}+R_{1 r}-I\left(U, U_{2}, X_{1} ; Y_{1}\right)+4 \epsilon\right)} \\
& +2^{n\left(R_{2 c}+R_{2 r}-I\left(U, U_{2}, X_{1} ; Y_{1}\right)+4 \epsilon\right)}
\end{aligned}
$$

Now, from (19) and (21), given that $I\left(U_{i} ; Y_{j} \mid X_{j}, U\right)<$ $I\left(U, U_{i}, X_{j} ; Y_{j}\right)$, we have that the probability of error due to (ii) can be made arbitrarily small if the following conditions hold:

$$
\left\{\begin{aligned}
R_{2 c}+R_{2 r} & \leqslant I\left(U_{2} ; Y_{1} \mid X_{1}, U\right) \\
R_{1 p} & \leqslant I\left(X_{1} ; Y_{1} \mid U, U_{1}, U_{2}\right) \\
R_{1 c}+R_{1 r}+R_{1 p}+R_{2 c}+R_{2 r} & \leqslant I\left(U, U_{2}, X_{1} ; Y_{1}\right) .
\end{aligned}\right.
$$

The same analysis is carried out for transmitter 2 and we obtain the following conditions:

$$
\left\{\begin{aligned}
R_{1 c}+R_{1 r} & \leqslant I\left(U_{1} ; Y_{2} \mid X_{2}, U\right) \\
R_{2 p} & \leqslant I\left(X_{2} ; Y_{2} \mid U, U_{1}, U_{2}\right) \\
R_{2 c}+R_{2 r}+R_{2 p}+R_{1 c}+R_{1 r} & \leqslant I\left(U, U_{1}, X_{2} ; Y_{2}\right) .
\end{aligned}\right.
$$

From the probability of error analysis, we have that the rate-pairs achievable with the proposed randomized coding scheme with feedback are those simultaneously satisfying conditions (22) and (23). Indeed, when $R_{1 r}=R_{2 r}=0$, the coding scheme described above reduces to the coding scheme presented in [10] and the achievable region corresponds to the entire capacity region of $\mathcal{C}_{\mathrm{FB}}$. In terms of the linear deterministic model, we have that such an achievable region can be characterized as follows.

Lemma 5: The achievable region of the randomized coding scheme with feedback in the linear deterministic IC is the set 
of pairs $\left(R_{1 c}, R_{1 r}, R_{1 p}, R_{2 c}, R_{2 r}, R_{2 p}\right)$ that satisfy, for all $i \in$ $\{1,2\}$,

$$
\begin{cases}R_{i c}+R_{i r} & \leqslant n_{j i} \\ R_{j p} & \leqslant\left(n_{j j}-n_{i j}\right)^{+} \\ R_{i c}+R_{i r}+R_{i p}+R_{j c}+R_{j r} & \leqslant \max \left(n_{i i}, n_{i j}\right) .\end{cases}
$$

In the following, we re-write the achievable rates using the randomized coding scheme in terms of $R_{1}=R_{1 p}+R_{1 c}$ and $R_{2}=R_{2 p}+R_{2 c}$. This yields

$$
\left\{\begin{array}{l}
R_{1}+R_{1 r} \leqslant \max \left(n_{11}, n_{21}\right) \\
R_{1}+R_{1 r}+R_{2 r} \leqslant \max \left(n_{11}, n_{12}\right) \\
R_{2}+R_{2 r} \leqslant \max \left(n_{22}, n_{12}\right) \\
R_{2}+R_{2 r}+R_{1 r} \\
R_{1}+R_{1 r}+R_{2}+R_{2 r} \leqslant \max \left(n_{22}, n_{21}\right)
\end{array}\right.
$$

with $a=\max \left(n_{11}, n_{12}\right)+\left(n_{22}-n_{12}\right)^{+}$and $b=$ $\max \left(n_{22}, n_{21}\right)+\left(n_{11}-n_{21}\right)^{+}$. Indeed, the region characterized by (25) corresponds to the region $\mathcal{C}_{\mathrm{FB}}$ when $R_{1 c}=R_{2 c}=0$. This implies that it is always possible to achieve any rate pair $\left(R_{1}, R_{2}\right) \in \mathcal{C}_{\mathrm{FB}}$ by using the randomized coding scheme and properly choosing the random common rates $R_{1 c}$ and $R_{2 c}$.

On another note, we highlight the fact that any rate pair $\left(R_{1 c}, R_{1 r}, R_{1 p}, R_{2 c}, R_{2 r}, R_{2 p}\right) \in \mathcal{C}_{\mathrm{FB}} \cap \mathcal{B}_{\mathrm{FB}}$ satisfies for all $i \in\{1,2\}$,

$$
R_{i c}+R_{i r}<n_{j i}
$$

This implies that, when using the randomized coding scheme with feedback presented above, transmitter $i$ can always reliably decode the common message and common random message $w_{j}^{(b)}$ from receiver $j$ using feedback at the end of block $b$. This observation follows immediately from substituting the condition $R_{i p}+R_{i c} \geqslant L_{i}, \forall i \in\{1,2\}$ into the last inequality of (24).

In the following, we describe the notion of saturation in the context of the rate pairs in $\mathcal{C}_{\mathrm{FB}}$.

Definition 3 (Saturation): $A$ rate pair $\left(R_{1 c}, R_{1 r}, R_{1 p}, R_{2 c}, R_{2 r}, R_{2 p}\right) \in \mathcal{C}_{\mathrm{FB}}$ is said to be saturated if it satisfies the following conditions for all $i \in\{1,2\}$ :

$$
\begin{cases}R_{i c}+R_{i r} & \leqslant n_{j i} \\ R_{j p} & \leqslant\left(n_{j j}-n_{i j}\right)^{+} \\ R_{i c}+R_{i r}+R_{i p}+R_{j c}+R_{j r} & =\max \left(n_{i i}, n_{i j}\right) .\end{cases}
$$

The main feature of a saturated rate pair $\left(R_{1 c}, R_{1 r}, R_{1 p}, R_{2 c}, R_{2 r}, R_{2 p}\right)$ is that if transmitter $i$ unilaterally deviates by increasing its individual rate, i.e., adopting other rates $R_{i c}^{\prime}, R_{i r}^{\prime}$ and $R_{i p}^{\prime}$, with $R_{1 p}^{\prime}+R_{1 c}^{\prime}>R_{1 p}+R_{1 c}$, then, receiver $i$ cannot reliably decode the information with an arbitrarily small probability of error. That is, given the rates $R_{1 r}$ and $R_{2 r}$, the new rate pair $\left(R_{i p}^{\prime}+R_{i c}^{\prime}, R_{2 p}+R_{2 c}\right)$ is not in the achievable region of the randomized coding scheme with feedback characterized in (25).

In the following, we show that any saturated rate pair $\left(R_{1 c}, R_{1 r}, R_{1 p}, R_{2 c}, R_{2 r}, R_{2 p}\right) \in \mathcal{C}_{\mathrm{FB}}$ is achievable at an NE. Lemma 6: Any saturated rate pair $\left(R_{1 c}, R_{1 r}, R_{1 p}, R_{2 c}, R_{2 r}, R_{2 p}\right) \in \mathcal{C}_{\mathrm{FB}}$ satisfies the condition that $\left(R_{1 c}+R_{1 p}, R_{2 c}+R_{2 p}\right) \in \mathcal{C}_{\mathrm{FB} / \mathrm{NE}}$.

Proof: From Lemma 5, we know that there exists a coding scheme with feedback that achieves a rate $\boldsymbol{R}=\left(R_{1 c}-\right.$ $\left.\frac{\eta}{6}, R_{1 r}-\frac{\eta}{6}, R_{1 p}-\frac{\eta}{6}, R_{2 c}-\frac{\eta}{6}, R_{2 r}-\frac{\eta}{6}, R_{2 p}-\frac{\eta}{6}\right)$ for all $\eta>0$.
Thus, transmitters experience the rates $R_{1}=R_{1 p}+R_{1 c}-\frac{\eta}{3}$ and $R_{2}=R_{2 p}+R_{2 c}-\frac{\eta}{3}$. Let us assume now that such coding schemes with feedback are not $\eta$-equilibria. Then, there must exist another coding scheme for transmitter $i$, not necessarily a coding scheme with feedback and with possibly a different code length $n^{\prime}$, that improves the rate $R_{i}$ by at least $\eta$. Without any loss of generality, assume that transmitter 1 is the deviating transmitter. Then, after the deviation from $\boldsymbol{R}$, transmitter 1 achieves a new rate $\tilde{R}_{1}>R_{1}+\eta$. The set of achievable rate pairs $\left(\tilde{R}_{1}, R_{2 p}+R_{2 c}\right)$ obtained when transmitter 1 unilaterally deviates is described by

$$
\left\{\begin{array}{ll}
\tilde{R}_{1}+R_{1 r} & \leqslant \max \left(n_{21}, n_{11}\right) \\
\tilde{R}_{1}+R_{1 r}+R_{2 p}+R_{2 c}+R_{2 r} & \leqslant \min (a, b) \\
R_{2 c}+R_{2 r} & \leqslant n_{12} \\
\tilde{R}_{1}+R_{1 r}+R_{2 c}+R_{2 r} & \leqslant \max \left(n_{11}, n_{12}\right)
\end{array},\right.
$$

where $a$ and $b$ are the same as in (25). The conditions in (28) are obtained by rewriting the conditions in (24) in terms of $\tilde{R}_{1}, R_{2 p}, R_{2 c}, R_{2 r}$ and $R_{1 r}$.

However, from the assumption that the rate pair $\boldsymbol{R}$ is saturated, increasing $R_{1}$ by $\eta$ must lead the rate pair $\left(\tilde{R}_{1}, R_{2 p}+\right.$ $R_{2 c}$ ) to violate at least the last condition in (28). Note that the first two conditions in (28) are satisfied by the assumption that the rate $\tilde{R}_{1}$ is achievable. The third inequality holds because only transmitter 1 has deviated from the rate pair $\boldsymbol{R}$ and the previous rate pair $\left(R_{1 p}+R_{1 c}, R_{2 p}+R_{2 c}\right)$ was achievable. We verify whether the last inequality holds. First, we ignore the term $R_{1 c}$ as the random symbols are assumed to be known at receiver 1 and thus, they do not convey any new information to receiver 1 . Moreover, the new strategy of transmitter 1 might not use random symbols. Then, we write,

$$
\begin{aligned}
n\left(\tilde{R}_{1}+R_{2 c}+R_{2 r}\right)= & H\left(W_{1}, W_{2 c}, W_{2 r}\right) \\
\stackrel{(a)}{=} & H\left(W_{1}, W_{2 c}, W_{2 r} \mid W_{1 r}\right) \\
= & I\left(W_{1}, W_{2 c}, W_{2 r} ; Y_{1}^{n} \mid W_{1 r}\right) \\
& +H\left(W_{1}, W_{2 c}, W_{2 r} \mid Y_{1}^{n}, W_{1 r}\right) \\
= & I\left(W_{1}, W_{2 c}, W_{2 r} ; Y_{1}^{n} \mid W_{1 r}\right) \\
& +H\left(W_{1} \mid Y_{1}^{n}, W_{1 r}\right) \\
& +H\left(W_{2 c}, W_{2 r} \mid Y_{1}^{n}, W_{1}, W_{1 r}\right) \\
& (\text { b) } \quad \\
\leqslant & I\left(W_{1}, W_{2 c}, W_{2 r} ; Y_{1}^{n} \mid W_{1 r}\right) \\
& +\delta_{n} \\
& +H\left(W_{2 c}, W_{2 r} \mid Y_{1}^{n}, W_{1}, W_{1 r}, X_{1}^{n}\right) \\
& \\
& n \max \left(n_{11}, n_{12}\right)+\delta_{n},
\end{aligned}
$$

where, $(a)$ follows from the independence of the random messages $w_{1 r}$ (if they exist) from messages $w_{1}, w_{2 c}$ and $w_{2 r}$; (b) follows from Fano's inequality; and (c) follows from the fact that common and common random messages $w_{2 c}$ and $w_{2 r}$ become deterministic if $\boldsymbol{y}_{1}^{n}, w_{1}$ and $w_{1 r}$ are known at receiver 1. Thus, $H\left(W_{2 c}, W_{2 r} \mid Y_{1}^{n}, W_{1}, W_{1 r}, X_{1}^{n}\right)=0$.

Now, since $\delta_{n}$ can be made arbitrarily small such that $\delta_{n}<$ $\frac{\eta}{3}$, it follows that

$$
\tilde{R}_{1}+R_{2 c}+R_{2 r} \leqslant \max \left(n_{11}, n_{12}\right)+\frac{\eta}{3},
$$

and there is no violation to the fourth condition in (28). Thus, since no violation is verified, this contradicts the assumption 
that the rate pair $\boldsymbol{R}$ is saturated. Thus, this deviation cannot exist and $\left(R_{1 c}+R_{1 p}, R_{2 c}+R_{2 p}\right) \in \mathcal{C}_{\mathrm{FB} / \mathrm{NE}}$, which completes the proof.

We have seen that every self-saturated rate pair $\left(R_{1 c}, R_{1 c}, R_{1 p}, R_{2 c}, R_{2 r}, R_{2 p}\right)$ is an $\eta$-equilibrium. Then, we finalize the proof by proving the following lemma.

Lemma 7: For all $\left(R_{1}, R_{2}\right) \in \mathcal{C}_{\mathrm{FB}} \cap \mathcal{B}_{\mathrm{FB}}$, there always exists an achievable rate pair $\left(R_{1 c}, R_{1 r}, R_{1 p}, R_{2 c}, R_{2 r}, R_{2 p}\right)$, with $R_{1}=R_{1 p}+R_{1 c}$ and $R_{1}=R_{1 p}+R_{1 c}$ that is saturated.

Proof: A rate pair $\left(R_{1 c}, R_{1 r}, R_{1 p}, R_{2 c}, R_{2 r}, R_{2 p}\right)$ that is saturated, with $R_{i c}+R_{i p} \geqslant L_{i}$, satisfies the following conditions:

$$
\begin{cases}R_{1 c}+R_{1 p} & \geqslant\left(n_{11}-n_{11}\right)^{+} \\ R_{1 p} & \leqslant\left(n_{11}-n_{11}\right)^{+} \\ R_{1 c}+R_{1 p}+R_{1 r}+R_{2 c}+R_{2 r} & =\max \left(n_{11}, n_{12}\right) \\ R_{2 c}+R_{2 p} & \geqslant\left(n_{11}-n_{11}\right)^{+} \\ R_{2 p} & \leqslant\left(n_{11}-n_{11}\right)^{+} \\ R_{2 c}+R_{2 p}+R_{2 r}+R_{1 c}+R_{1 r} & =\max \left(n_{22}, n_{21}\right) .\end{cases}
$$

We write the region characterized by (30) in terms of $R_{1}=$ $R_{1 c}+R_{1 p}$ and $R_{2}=R_{2 c}+R_{2 p}$. This yields,

$$
\begin{cases}R_{1} & \geqslant\left(n_{11}-n_{12}\right)^{+} \\ R_{2} & \geqslant\left(n_{22}-n_{21}\right)^{+} \\ R_{1}+R_{1 r}+R_{2}+R_{2 r} & \leqslant \min (a, b),\end{cases}
$$

where $a$ and $b$ are the same as in (25). Note that the region described by (31) corresponds exactly to the region $\mathcal{C}_{\mathrm{FB}} \cap \mathcal{B}_{\mathrm{FB}}$. This implies that for any pair $\left(R_{1}, R_{2}\right) \in$ $\mathcal{C}_{\mathrm{FB}} \cap \mathcal{B}_{\mathrm{FB}}$, there always exists a saturated rate pair $\left(R_{1 c}, R_{1 r}, R_{1 p}, R_{2 c}, R_{2 r}, R_{2 p}\right)$ that is an NE and achievable with a randomized coding scheme with feedback. This completes the proof.

Finally, the proof of Theorem 1 is immediate from Lemma 5, Lemma 6 and Lemma 7.

\section{Conclusions}

In this paper, the Nash equilibrium region of the two-user linear deterministic interference channel with feedback has been studied when transmitters aim to selfishly maximize their individual achievable rates by autonomously tuning their own transmit configurations. That is, the set of all achievable rates that can be observed at a NE has been fully characterized. In this work, a transmit configuration refers to the set of parameters such as the number of information bits per block, block length, codebook and encoder/decoder function. One of the most interesting observations presented in this paper is that, in all the interference regimes, the use of feedback increases the number of rate pairs that are achievable at the NE with respect to the case without feedback. More importantly, the new achievable rate pairs are either Pareto optimal or weakly Pareto superior to the rate pairs achievable without feedback at the NE. Surprisingly, another important observation is that using feedback all Pareto optimal rate pairs of the capacity region of the LD-IC with feedback, that is, all the sumrate maximizing pairs, are achievable at an $\mathrm{NE}$, at least in the symmetric case as we showed in several examples. This strongly contrasts with the case without feedback, where only a subset of the Pareto optimal rate pairs of the capacity region of the LD-IC without feedback are achievable at the equilibrium in most of the interference regimes.

\section{REFERENCES}

[1] S. Avestimehr, S. Diggavi, and D. N. C. Tse, "Wireless network information flow," in Proceedings of the 45th Annual Allerton Conference on Communication, Control and Computing, Monticello, IL, Sep. 2007.

[2] - "Wireless network information flow: A deterministic approach," IEEE Transactions on Information Theory, vol. 57, no. 4, pp. 1872 1905, Apr. 2011.

[3] R. A. Berry and D. N. C. Tse, "Shannon meets Nash on the interference channel," IEEE Transactions on Information Theory, vol. 57, no. 5, pp 2821-2836, May 2011.

[4] G. Bresler and D. N. C. Tse, "The two user Gaussian interference channel: A deterministic view," European Transactions on Telecommunications, vol. 19, no. 4, pp. 333-354, Apr. 2008.

[5] A. El Gamal and M. Costa, "The capacity region of a class of deterministic interference channels," IEEE Transactions on Information Theory, vol. 28, no. 2, pp. 343-346, Mar. 1982.

[6] R. H. Etkin, D. N. C. Tse, and W. Hua, "Gaussian interference channel capacity to within one bit," IEEE Transactions on Information Theory, vol. 54, no. 12, pp. 5534-5562, Dec. 2008.

[7] J. F. Nash, "Equilibrium points in n-person games," Proceedings of the National Academy of Sciences of the United States of America, vol. 36, no. 1, pp. 48-49, Jan. 1950.

[8] S. M. Perlaza, "Game theoretic approaches to spectrum sharing in decentralized self-configuring networks," Ph.D. dissertation, Télécom ParisTech, Jul. 2011.

[9] L. Rose, S. M. Perlaza, and M. Debbah, "On the Nash equilibria in decentralized parallel interference channels," in Proceedings of the IEEE International Conference on Communications (ICC), Kyoto, Japan, Jun. 2011.

[10] C. Suh and D. N. C. Tse, "Feedback capacity of the Gaussian interference channel to within 2 bits," IEEE Transactions on Information Theory, vol. 57, no. 5, pp. 2667-2685, May 2011. 